chapter on amyloid which is good to see and much better than in the first edition but it is a pity that the Sulphate Alcian Blue method is dismissed, for although not specific, it is a most useful method for screening. It is also surprising that dextrin sucrose mountant is not mentioned, as this effectively prevents 'bleeding' in metachromatic methods.

The sections on pigments and minerals are good, having been completely revised and expanded. Each now has a separate chapter devoted to it. Those on enzyme histochemistry have been expanded, brought up to date and new chapters included on the principles of enzyme histochemistry and on ultrahistochemistry.

This is a good bench book for anyone with an interest in practical histochemistry and is very suitable for H.N.C. students. The methods are clearly and concisely laid out and only one major error detected, Method 11 omits to turn on the cooling water! When getting down to the detail and theory of individual methods, especially in enzyme histochemistry, the text needs support from a standard histochemistry text book or from the good references included at the end of each chapter but, as a practical manual, the book amply fulfils its purpose. Maybe a little more of the author's experience of possible pitfalls could have been included in the text, for example, in methods 18 and 25 , the aluminium chloride and paraldehyde must be fresh. It is also a pity that for adenosine triphosphatase only a magnesium activated method is given since this is not suitable for myosin adenosine triphosphatase. A calcium activated method should have been included.

Minor criticisms apart, this is an excellent manual which deserves a place in every histology laboratory.

\section{An Outline of Geriatrics}

\section{(Monographs for Students of Medicine)}

By H. M. Hodkinson. Pp. xiii +159 , soft cover. Academic

Press, London, New York, San Francisco, 1975. £2.40.

This is one of several monographs for students of medicine in which the author makes it clear that he is hoping it will appeal also to a wider circle of doctors, including general practitioners and other personnel involved with the rehabilitation of the elderly. In a short book such as this the author had to be necessarily selective in the choice of subjects. However, general aspects and principles are dealt with reasonably fully but specific topics are described succinctly. The twenty-three chapters give a 'bird's-eye view' of the whole field of medicine of old age. Those ending with the author's own conclusions were very helpful in focusing one's attention on important points. The students will find the guide to history-taking and investigation most useful. Moreover, the pitfals of over-investigating old people and incorrectly interpreting results are emphasized and well discussed in the chapter on 'Biochemical Investigation'.

However, what the students will find particularly of value in the monograph are the sections on rehabilitation, nursing care and drug therapy. Chapters on problems such as incontinence, constipation and pressure sores are given, and rightly so, more space and contain sound advice regarding prevention and treatment. Long stay and irremediable patients have not been forgotten and we all would agree with the author's plea for compassion and understanding rather than scientific procedures in the care of the terminally ill.

The section on mental disturbance in old age is short but a list of up-to-date references will help those who wish to read further. The omission, in the discussion on the social component of geriatrics, of some of the marital difficulties and psycho-sexual problems in the elderly, is regretted.

Finally, it is to be hoped that the author's enthusiasm for geriatrics will generate the same in the medical students and encourage them to take up the speciality.
Planning a Course of Continuing Education for General Practice-A Systematic Approach

By Roger B. Cole and Charles E. Engel. Pp. $v+42$, soft cover, illustrated. London: Dept of Audio Visual Communication B.M.A., 1975.

The value of this short study lies not so much in its content as in its approach. The content concerns 'paediatric cardiology for general practitioners'. The intended learner is a general practitioner practising in rural Illinois, whose undergraduate medical education was completed at least 5 years ago. It is assumed that there is no local access to a specialist in the field. Finally, the patient age-range is defined as 15 years to 4 weeks: the reason for the lower limit is that most women in Illinois are delivered in hospital, where there is access to paediatric consultation for the critically ill newborn. It is therefore assumed, in this educational programme, that the majority of infants with critical heart lesions will have been recognized during the first 4 weeks of life.

Much more important than the details of content is the soundness of the educational approach. The classification of objectives used here into 'understanding and knowledge' and 'competences' is particularly agreeable. This seems to be much more useful and simple than the more elaborate Bloom's taxonomy and at the same time more readily applicable to the experience of clinical medicine than 'knowledge, skills and attitudes'.

The programme is presented as a series of broad goals with a number of enabling objectives and each of these is further sub-divided into its component parts, each with specific behaviourally worded objectives related to understanding and knowledge, and related to competence. The detail is obsessive and many of the enabling objectives related to understanding and knowledge seem to be quite unnecessary in terms of the related competence. For example, the general practitioner is expected to recognize a potential 'alerter' (that is to say, symptom which might suggest the presence of a congenital heart lesion). One of these is given as 'irritable or fussy baby', and an enabling objective related to understanding and knowledge, that the general practitioner will be able to explain in physiological terms why irritability is often associated with large left to right shunts at the ductal and ventricular levels and cyanotic lesions with or without congestive heart disease. It is true that these enabling objectives are usually labelled as being desirable but not essential for the learner. The focus of the programme is on competence, but the precise relationship between the stated competences and some of the quite detailed enabling objectives relating to understanding and knowledge, often requiring physiological explanations, is not explored.

In the past few years it has become increasingly clear that the problems of continuing education for general practitioners are among the most challenging for medical education. Increasingly the emphasis will shift towards defining the necessary competences and then towards assessment of those competences by the learner as part of his own learning process. The approach taken in this book is therefore a prerequisite for clinical audit.

The last part of the book describes a 'twenty-minute armchair' problem. The learner is presented with a 2-month-old male infant with rapid and laboured breathing. He is then allowed to choose from among a small number of management decisions, and by turning the relevant page to read a comment on his decision, or to be given further information on which he is enabled to make further choices. This is another version of problem-oriented teaching, similar to that used by the RCGP in the modified essay question.

This book will be of particular interest not only to those concerned with the continuing education of general practioners, but to course organizers of vocational training schemes. 